\title{
Steuerung im Gesundheitswesen: zuviel, zuwenig oder falsch?
}

\author{
P. Berchtold ${ }^{a}$ K. Hess ${ }^{b}$
}

Wieviel Steuerung braucht das Gesundheitswesen? Und wieviel vertragen seine Akteure? Wer soll mit welchen Instrumenten wen steuern? Ist ärztliche Budgetverantwortung unethisch? Das erste Symposium des neuen Forums Managed Care vom 25. Mai 2005 im World Trade Center Zürich setzt sich mit diesen Fragen auseinander. Referenten aus allen Bereichen des Gesundheitssystems werden sich in Plenarveranstaltungen, Workshops und Diskussionsgruppen mit den aktuellen Fragen des Managed Care auseinandersetzen.

\footnotetext{
a Präsident Forum Managed Care, Leiter College-M

b Vizepräsident Forum Managed Care
}

Korrespondenz:

PD Dr. Peter Berchtold

College für Management

im Gesundheitswesen

Freiburgstrasse 41

CH-3010 Bern

E-Mail:

peter.berchtold@college-m.ch

Dr. med. et lic. oec. Kurt Hess Beratungen und Projekte

im Gesundheitswesen

Grossmünsterplatz 1

CH-8001 Zürich

E-Mail: kurt.hess@hin.ch
Managed Care, in der Schweiz noch kaum anderthalb Jahrzehnte alt, ist zu einem gesundheitspolitischen Thema ersten Ranges avanciert. Bundesrat, Kommissionen und die eidgenössischen Räte beschäftigen sich mit richtungsweisenden Entscheidungen, bei welchen den Modellen und Instrumenten des Managed Care eine zentrale Rolle zufällt. Doch welche Steuerungskonzepte eignen sich für schweizerische Verhältnisse, welche Anreizsysteme führen über die marktwirtschaftlichen Prinzipien zur Gesundung des Gesundheitswesens? Während die einen Managed Care als unzulässigen Eingriff in die ärztliche Freiheit verteufeln, erwarten andere wahre Wunder von dessen Systemen und Instrumenten. Beide Haltungen zielen an der Realität vorbei. Mangels klarer, generalisierbarer und auf die Schweiz übertragbarer Evidenzen bewegt sich die aktuelle Diskussion um Managed Care vorwiegend im Bereich der Anekdoten, Behauptungen, Interessenproklamationen und Mutmassungen. Trotz dieses Mangels kristallisiert sich heute kaum mehr ernsthaft bestritten - ein substantielles Potential dieser neuen Prinzipien für eine nutzen- und patientenorientierte medizinische Versorgung heraus. Weshalb also soll man Managed Care nicht grossflächig lancieren?

Man könnte auch fragen: Wer hat eigentlich den Anreiz, die Anreize zu verändern? Für die meisten Akteure sind sie heute durchaus vorteilhaft. Es gilt also vordringlich, Transparenz, Akzeptanz und Engagement (oder wenigstens Neugier!) für die neuen Versorgungsmodelle und das vielfältige Steuerungsinstrumentarium zu schaffen. Die Konsequenzen des sukzessiven Systemwechsels sind bei vielen Leistungserbringenden wenig beliebt, weshalb sich diese vielerorts dem Vorwurf des Verhinderns und der
Kooperationsunfähigkeit ausgesetzt sehen. Das erstaunt wenig, denn Steuerung und Management - in welcher Form auch immer - beschneiden den professionellen Gestaltungsraum der Ärzte, Pflegenden und Angehörigen anderer Gesundheitsberufe in ihrer zentralen Kompetenz, der Behandlung und Betreuung von Patienten [1]. Natürlich kommt aber auch dieses Kerngeschäft, das in den vergangenen Jahren durch Neuerungen und Innovationen sehr viel komplexer geworden ist, nicht ohne ein Mehr an aktivem Management und Steuerung aus. Verwalten und Gestalten reichen nicht mehr, zu viele Personen und Funktionen sind in den Behandlungsketten involviert.

Das Gesundheitswesen braucht so gesehen nicht nur mehr Steuerung, sondern richtige, berufsbezogene, gerechte, zielorientierte und vor allem auch konzertierte Steuerung. Es liegt in der Natur von Management und Managed Care, «transparente Verantwortlichkeit» (englisch accountability) zu fordern, denn nur auf dieser Basis kann gesteuert und gemanagt werden. Auf der Ebene der Leistungserbringenden kollidiert eine solche Forderung aber mit der traditionellen Expertenautonomie, z.B. von jenen Ärzten, die grundsätzlich und ausschliesslich eine Verantwortlichkeit anerkennen - jene gegenüber dem individuellen Patienten.

\section{Neue Anreizstrukturen tun not}

Der anstehende Paradigmenwechsel führt zur Frage, wie sich Leistungserbringende in weitergehende Steuerungsmassnahmen im Sinn von Managed Care und integrierter Versorgung einbinden lassen. Gelingt dies nicht, drohen Polarisierung und Konfrontation, welche die heute noch vorherrschende Abwertung von Managed Care als qualitativ schlechtere Billigmedizin festigen können. Dass sich Managed Care auf der politischen Ebene heute einer steigenden Beliebtheit erfreut, ist $\mathrm{zu}$ begrüssen, ändert am zweifelhaften Image aber wenig. Die Einführung von Managed-Care-Modellen und -Instrumenten zu fördern, heisst einen kulturellen Wandel voranzutreiben [2]. Dazu eignet sich aber kaum 
ein rigoroser Top-down-Ansatz in Form von Gesetzen und Vorschriften, ein Wandel kann nachhaltig nur auf der Grundlage von geeigneten Anreizstrukturen vollzogen werden, die den Beteiligten einen Nutzen und dem Gesamtsystem eine marktwirtschaftliche Basis bringen. Neue Anreize dürfen nicht nur finanzieller Art sein, denn damit wird - wie wir in den vergangenen Jahren erfahren haben - das Vorurteil der Billigmedizin gestärkt und eine breitere Akzeptanz von Managed Care vor allem bei den Patienten verhindert. Sinnstiftende Anreize bewirken, dass

- Versorgungsleistungen nicht nur mit Preisschildern, sondern auch mit expliziten Qualitätslabeln versehen sind;

- die Eigenverantwortlichkeit der Patienten gestärkt wird;

- neue, attraktive Qualifizierungswege und Tätigkeitsfelder für Gesundheitsberufe entstehen;

- administrative Tätigkeiten vereinfacht werden;

- Managed-Care-Konzepte und -Instrumente wissenschaftlich auf ihre Wirksamkeit, Zweckmässigkeit und Wirtschaftlichkeit untersucht werden.

\section{Ganz ohne finanzielle Anreize geht es nicht}

So wenig die finanziellen Anreize dominieren sollen, so unbestritten ist ihre Wirksamkeit. Eine entscheidende Rolle spielt dabei das Vergütungssystem. Die heute weit überwiegend praktizierte Einzelleistungsvergütung (fee for service) sei unethisch, sagen die einen, weil sie den Anreiz zur Überversorgung und zu unnötigen Leistungen in sich trage. Pauschalvergütungen und insbesondere Kopfpauschalen (capitation) führten zu Unterversorgung und zum Vorenthalten von Leistungen und seien deshalb unethisch, sagen die andern. Dass Vergütungsformen das Verhalten von Ärzten beeinflussen, wissen wir spätestens aus einem kürzlich erschienenen Review der Cochrane Collaboration, deren systematische Übersichtsarbeiten als Evidenzgoldstandard im Gesundheitsbereich gelten: Bei «fee for service» fanden die Autoren signifikant mehr Konsultationen und mehr spezialisierte Abklärungen und Behandlungen als bei pauschaler Vergütung [3]. Ob dieses Mehr - und auf der anderen Seite das Weniger - für den individuellen Patienten gut oder schlecht ist, lässt sich auch mit aufwendigen statistischen Analysen kaum belegen. Für pauschalierte Vergütungsformen ist es deshalb wichtig, dass sie sich innerhalb eines festgelegten Rahmens bewegen, z. B. indem
- Budgetmitverantwortung nur mit Ärztegruppen (und nicht mit den einzelnen Ärzten) vereinbart wird;

- explizite Qualitätsausweise (Zertifizierungen) gefördert und gefordert werden;

- Patienten transparent informiert werden;

- der Zugang zu Zweitmeinungen gewährleistet wird;

- der Risikoausgleich verbessert wird.

Keiner der Akteure hat im Ernst ein Interesse an qualitativ schlecht behandelten und unterversorgten Patienten. Trotzdem ist es legitim, dass z.B. Versicherer und staatliche Kostenträger nach möglichst günstigen Preisen - bei nachgewiesenem Nutzen, versteht sich - für Behandlung und Betreuung trachten. Sie entsprechen damit einer Erwartung der Prämien- und Steuerzahlenden. Genauso wie Patienten von ihren behandelnden Ärzten und Pflegenden erwarten, dass sie nichts Notwendiges unterlassen, muss umgekehrt erwartet werden, dass Versicherer und Staat nach den Prinzipien der Wirtschaftlichkeit handeln. Diese Divergenz der Interessen akzentuiert sich, wenn die Finanzmittel knapp werden. Rahmenbedingungen, die für alle gelten, sollen gewährleisten, dass die Divergenz nicht zur lähmenden Konfrontation verkommt. Und sie sollen Gestaltungsräume abstecken, z. B., um die Frage zu beantworten, was wir unter medizinischer Notwendigkeit oder unter Minimalstandards für die Behandlung und Betreuung verstehen wollen. Der Ruf nach Ethik wird lauter und zeigt, wie dringlich die genannte Frage ist [4]. Und er macht gleichzeitig klar, dass diese Frage nicht einseitig vom einen oder anderen Akteur, sondern nur im offenen Dialog sinnvoll angegangen werden kann. Dazu will das Forum Managed Care mit seinem diesjährigen Symposium vom 25. Mai 2005 einladen.

\section{Literatur}

1 Berchtold P, Schmitz C. (Un)happy doctors? zwischen Arzt-Sein und Managen. Schweiz Ärztezeitung 2005;86(6):339-40.

2 Finsterwald D. Managed Care - Pionierland Schweiz. SGGP-Schriftenreihe 2004, Band 75.

3 Gosden T, Forland F, Kristiansen IS, Sutton M, Leese B, Giuffrida A, et al. Capitation, salary, fee-for-service and mixed systems of payment: effects on the behaviour of primary care physicians (Cochrane Review). In: The Cochrane Library. Issue 3. Chichester: John Wiley \& Sons, Ltd.; 2004.

4 Povar GJ, Blumen H, Daniel J, Daub S, Evans L, Holm RP, et al. Ethics in practice: managed care and the changing health care environment. Medicine as a profession managed care ethics working group statement. Ann Intern Med 2004;141:131-6. 